\title{
AVALIAÇÃO DOS EFEITOS DA SUPLEMENTAÇÃO COM VITAMINAS E E C SOBRE OS ÁCINOS DA GLÂNDULA PARÓTIDA DE RATOS DIABÉTICOS CRÔNICOS: ANÁLISE MORFOLÓGICA E QUANTITATIVA
}

\author{
ASSESSMENT OF THE EFFECTS OF VITAMINS E AND C SUPPLEMENTATION ON THE PAROTID \\ GLAND ACINUS OF CHRONIC DIABETIC RATS: MORPHOLOGICAL AND QUANTITATIVE ANALYSIS
}

\section{Ângela Maria Pereira Alves ${ }^{a}$, Éder Paulo Belato Alves ${ }^{b}$, Josiane Medeiros de Melloc, Jacqueline Nelisis Zanoni ${ }^{\mathrm{d}}$, Danielle das Neves Bespalhoke, Cássio Rafael Moreira ${ }^{\mathfrak{f}}$ Fernanda Ferraris}

angela.01.com@gmail.com, bederpaulo.uem@gmail.com, cjmedeirosmello@gmail.com, djnzanoni@uem.br, edani_bespalhok@hotmail.com, ${ }^{\mathrm{f}} \mathrm{cassiorafamoreira@gmail.com}$, gferrarifernanda@yahoo.com.br Universidade Estadual de Maringá - Maringá (PR), Brasil

\section{RESUMO}

\begin{abstract}
Em animais e indivíduos diabéticos, são evidentes as alteraçôes na morfofisiologia das glândulas parótidas. Este trabalho teve por objetivo avaliar os efeitos da suplementação com vitaminas E e C sobre os parâmetros morfoquantitativos acinares da glândula parótida de ratos diabéticos induzidos por estreptozootocina. Vinte ratos machos foram distribuídos nos grupos normoglicêmico $(\mathrm{N})$, diabético $(\mathrm{D})$, normoglicêmico suplementado com vitaminas E e C (NEC) e diabético suplementado com vitaminas E e C (DEC). Aos 210 dias de idade, após eutanásia, foi extraída a parótida e foram feitos cortes histológicos submetidos à técnica de coloraçấo HE. Parâmetros fisiológicos, como massa corporal, consumos de água e ração e glicemia de jejum, foram analisados. Quantificaram-se todos os ácinos de 20 imagens. Verificou-se maior densidade acinar média no grupo NEC $(\mathrm{p}<0,05)$ em relação ao grupo $\mathrm{N}$. O mesmo resultado foi observado para o grupo DEC $(\mathrm{p}<0,05)$ em relação ao grupo $\mathrm{D}$. Foi mensurada a área $\left(\mu \mathrm{m}^{2}\right)$ de 500 ácinos em cada grupo estudado. Houve redução $(\mathrm{p}<0,05)$ na área acinar dos ratos dos grupos $\mathrm{D}$ e $\mathrm{DEC}$ em relaçáo ao grupo $\mathrm{N}$ e NEC. O grupo DEC apresentou maior área acinar média $(\mathrm{p}<0,05)$ quando comparado ao grupo $\mathrm{D}$. Com relação aos parâmetros morfoquantitativos, a suplementação com as vitaminas $\mathrm{E}$ e C foi positiva tanto para animais normoglicêmicos quanto para aqueles que apresentavam a condição de diabetes. Contudo, não houve alteraçóes significativas nos parâmetros fisiológicos de ratos com ou sem suplementação com as vitaminas $\mathrm{E}$ e C na comparação entre os grupos normoglicêmicos e entre os grupos diabéticos.
\end{abstract}

Palavras-chave: Glândula parótida; diabetes mellitus; vitamina E; vitamina C; suplementaçáo em ratos; ácinos.

\section{ABSTRACT}

In diabetic animals and patients, changes in the morphophysiology of the parotid glands are evident. This work aimed to evaluate the effects of supplementation with vitamins $\mathrm{E}$ and $\mathrm{C}$ on morphoquantitative acinar parameters of the parotid gland of diabetic rats induced by streptozotocin. Twenty male rats were divided into groups: normoglycemic $(N)$, diabetic $(D)$, normoglycemic supplemented with vitamins $E$ and $C$ (NEC) and diabetic supplemented with vitamins $\mathrm{E}$ and $\mathrm{C}$ (DEC). At 210 days of age, after euthanasia, the parotid was extracted and histological sections were made and stained through HE technique. Physiological parameters such as body weight, water and feed consumption, and fasting glucose were analyzed. Acini of 20 images were quantified. A higher acinar density average was noted in the NEC group $(\mathrm{p}<0.05)$ than in group N. The same result was observed for the DEC group $(\mathrm{p}<0.05)$ compared to group D. A 500 acini area $(\mu \mathrm{m} 2)$ was measured in each studied group. A reduction $(\mathrm{p}<0.05)$ in the acinar area of the rats in groups $\mathrm{D}$ and DEC was seen with respect to $\mathrm{N}$ and NEC groups. The DEC group had higher average acinar area $(\mathrm{p}<0.05)$ compared to group D. With respect to morphoquantitative parameters, supplementation with vitamins $\mathrm{E}$ and $\mathrm{C}$ was positive for both normoglycemic animals and those with diabetes. However, there were no significant changes in physiological parameters of rats with or without supplementation with vitamins $\mathrm{E}$ and $\mathrm{C}$, when comparing the normoglycemic and diabetic groups.

Keywords: Parotid gland; diabetes mellitus; vitamin E; vitamin C; supplementation in rats; acini. 


\section{Introdução}

No diabetes mellitus (DM), além das complicaçóes renais, oculares, nervosas, vasculares e cardíacas, longos períodos de hiperglicemia podem provocar alteraçóes bucais relevantes, como a doença periodontal, considerada a sexta complicação mais prevalente desta patologia ${ }^{1,2}$.

Em animais e indivíduos diabéticos, são notórias as alteraçôes na morfofisiologia das glândulas parótidas ${ }^{3}$. Essas alteraçóes têm como possíveis causas a baixa sensibilidade dos receptores muscarínicos da glândula parótida à acetilcolina ${ }^{4}$, mudanças no fluxo sanguíneo da glândula ${ }^{5}$, aumento da atividade autofágica e lisossomal, e endocitose de proteínas secretadas pelas células ductais $^{6}$. Isso reflete em redução do fluxo salivar e mudanças na composição da saliva, alteração no paladar, maior tendência à infecção bucal, deficiência no processo cicatricial, cáries, língua saburrosa, halitose, com consequente predisposiçáo à doença periodontal ${ }^{7}$.

Outro fator que contribui para o agravamento dessas alterações morfofisiológicas das glândulas salivares é o estresse oxidativo, que é intensificado pela condição de diabetes ${ }^{8}$.

O estresse oxidativo é caracterizado pelo aumento de espécies reativas de oxigênio (EROs) dentro da célula, e tem papel importante na patogênese das complicaçóes do $\mathrm{DM}^{9}$. Na glândula parótida, foram demonstradas alteraçóes em enzimas antioxidantes, como a catalase e a glutationa peroxidase ${ }^{10}$. Em consequência disso, as EROs podem ser produzidas por essas glândulas em situações de infecções microbianas e em resposta à hiperglicemia causada pelo $\mathrm{DM}^{11}$.

As vitaminas $\mathrm{E}$ e $\mathrm{C}$ exibem papel relevante na terapêutica das complicaçóes do diabetes e têm sido estudadas no tratamento das complicaçôes crônicas dessa patologia.

A vitamina $E$ ( $\alpha$-tocoferol) é um potente antioxidante lipofílico que neutraliza os radicais livres produzidos durante o metabolismo celular normal, protegendo as membranas celulares e lipoproteínas dos danos oxidativos $^{12}$. Além das propriedades antioxidantes, essa vitamina reduz a citotoxicidade, minimiza o efeito de lipoproteínas oxidadas, suprime a proliferação das células musculares lisas, reduz a adesão e agregação plaquetária e melhora a funçâo endotelial ${ }^{13}$.

A vitamina C (ácido ascórbico) tem um papel importante na proteçấo contra os danos celulares induzidos por radicais livres, além de regenerar a vitamina E. Foi constatada ${ }^{14}$ uma diminuição no nível basal de vitamina C no DM. A molécula dessa vitamina é estruturalmente similar à glicose, e pode substituí-la em muitas reações químicas, portanto é efetiva na prevenção da glicosilação não enzimática de proteínas ${ }^{15}$. Vários estudos têm demonstrado que o estresse oxidativo gerado pelo DM é um possível agente que afeta a estrutura e função da glândula salivar parótida. Considerando a importância das glândulas salivares no processo digestivo e na manutenção da saúde bucal, e sabendo da efetiva ação antioxidante das vitaminas $\mathrm{E}$ e $\mathrm{C}$ em vários modelos animais, o propósito deste trabalho foi avaliar os efeitos da suplementação com vitaminas $\mathrm{E}$ e $\mathrm{C}$ sobre os parâmetros morfoquantitativos acinares da glândula parótida de ratos diabéticos induzidos por estreptozootocina.

\section{Metodologia}

\section{Animais e indução do Diabetes Mellitus}

Foram utilizados 20 ratos machos Wistar (Rattus norvegicus), pesando em média 313 gramas, provenientes do Biotério Central da Universidade Estadual de Maringá, e a pesquisa foi aprovada pelo Comitê de Ética em Experimentação Animal daquela universidade, conforme parecer $n^{\circ}$ 018/2007. As técnicas utilizadas estão de acordo com princípios éticos adotados pelo Colégio Brasileiro de Experimentação Animal (COBEA).

Os ratos foram distribuídos em quatro grupos: $\mathrm{N}$ (normoglicêmico), NEC (normoglicêmico suplementado com vitaminas E e C), D (diabético), e DEC (diabético suplementado com vitaminas $\mathrm{E}$ e $\mathrm{C}$ ).

Aos 90 dias de idade, o DM foi induzido nos animais dos grupos D e DEC após jejum prévio de 14 horas, através de uma injeção intravenosa de estreptozootocina (35 mg por quilograma de massa corporal; Sigma, St. Louis, MO, EUA) dissolvida em uma solução tampão citrato $\mathrm{pH}$ 4,5 (10 mM). Posteriormente à indução, a glicemia dos animais foi determinada pelo método da glicose oxidase ${ }^{16}$ para confirmar o estabelecimento do modelo experimental.

Os animais dos grupos NEC e DEC receberam suplementação com vitaminas $\mathrm{E}$ e $\mathrm{C}$ por 120 dias. A vitamina E (Zhejiang NHU, China) foi incorporada na ração Nuvital (Nuvilab, Colombo, PR, Brasil) (1\% preparado a cada semana) e a vitamina C (Sigma, St. Louis, $\mathrm{MO}, \mathrm{EUA})$ foi adicionada à água $\left(1 \mathrm{~g} \mathrm{~L}^{-1}\right.$ diariamente preparado).

Os ratos foram mantidos em gaiolas individuais com fotoperíodo (12 horas de claro/escuro) e temperatura ambiente $\left(24^{\circ} \mathrm{C} \pm 2\right)$ controlados. A quantidade de alimento ingerido e a água consumida foram avaliadas durante a última semana de cada mês.

\section{Sacrifício, coleta e processamento do material}

Após 120 dias de experimento, os ratos foram previamente pesados e anestesiados com uma dose de 40 
$m g \mathrm{~kg}^{-1}$ de massa corporal de tiopental (Laboratórios Abbott, Chicago, IL, EUA) via intraperitoneal para realizar a coleta de sangue por punção cardíaca, com intuito de avaliar a glicemia de jejum e para a extração das glândulas, o que resultou na morte por exsanguinação.

As parótidas extraídas foram lavadas em solução salina $(\mathrm{NaCl} 0,9 \%)$ para retirada de resíduos de sangue e transferidas para solução de formalina $10 \%$ por um período de 12 horas, promovendo a íntegra fixação dos tecidos glandulares. Sequencialmente, foram pesadas e lavadas em água corrente para serem armazenadas em álcool 70\%. Utilizando-se uma série crescente de concentraçóes de álcoois, os tecidos foram desidratados, diafanizados em xilol, incluídos em parafina e seccionados em micrótomo com $6 \mu \mathrm{m}$ de espessura. Posteriormente, os cortes foram submetidos à coloração em Hematoxilina-Eosina (HE).

\section{Análise morfológica e quantitativa dos ácinos glandulares}

As imagens dos ácinos foram capturadas por câmera de alta resoluçáo acoplada ao microscópio Olympus BX40, transmitidas para microcomputador e gravadas em CD. Por meio do programa computadorizado de análise de imagens Image-Pro Plus, foi mensurada a área $\left(\mu \mathrm{m}^{2}\right)$ do perfil de 100 ácinos por lâmina, perfazendo o total de 500 ácinos em cada grupo estudado, expressando-se os resultados em média das áreas dos ácinos por grupo.

Além disso, foram quantificados todos os ácinos de 20 imagens capturadas por animal em aumento de 20x, com a finalidade de delinear a densidade dos ácinos, sendo que os resultados foram expressos como número de ácinos por $\mathrm{mm}^{2}$ de glândula salivar parótida, perfazendo um total de 100 campos em uma área de $36 \mathrm{~mm}^{2}$ por grupo.

\section{Análise estatística}

Os dados coletados foram submetidos a testes estatísticos apropriados, como análise de variância e teste de Tukey, para comparação dos aspectos morfoquantitativos dos ácinos glandulares entre os grupos. O nível de significância foi de 5\%.

\section{Resultados}

Os ratos tornados diabéticos pela estreptozootocina (grupos D e DEC) apresentaram um quadro com características típicas da síndrome: poliúria, polidipsia, hiperfagia, hiperglicemia, ganho reduzido ou até mesmo perda de massa corporal.

No início do experimento, a massa corporal foi similar entre os grupos analisados. No entanto, ao final do experimento, a massa corporal dos ratos dos grupos $\mathrm{D}$ e DEC foram $25,8 \%$ e $35,2 \%$, respectivamente, menores em relação ao grupo N. A comparação entre os valores da massa corporal final entre os grupos $\mathrm{D}$ e $\mathrm{DEC}$ não mostrou diferença significativa (Tabela 1).

A média de consumo de ração dos ratos dos grupos $\mathrm{D}$ e DEC foi superior à dos ratos dos grupos N e NEC. A média da ingesta de água dos ratos dos grupos $\mathrm{D}$ e DEC não diferiram entre si, entretanto foram significativamente maiores que as verificadas para os ratos dos grupos $\mathrm{N}$ e NEC (Tabela 1).

Tabela 1: Massa corporal inicial com 90 dias de idade (MCl), massa corporal final com 210 dias de idade (MCF), ingesta de água por $100 \mathrm{~g}$ de peso corporal (IA), ingesta de ração por 100 g de peso corporal (IR), glicemia inicial (Gl) e glicemia final (GF) nos animais dos grupos normoglicêmico (N), normoglicêmico suplementado com vitaminas E e C (NEC), diabético (D) e diabético suplementado com vitaminas E e C (DEC).

\begin{tabular}{ccccc} 
Parâ- & \multicolumn{4}{c}{ Grupos } \\
metros & N & NEC & D & DEC \\
& & & & \\
MCI (g) & $304 \pm$ & $325,9 \pm$ & $316,6 \pm$ & $307,2 \pm$ \\
& $16,97 \mathrm{a}$ & $29,3 \mathrm{a}$ & $21,03 \mathrm{a}$ & $18,73 \mathrm{a}$ \\
MCF $(\mathrm{g})$ & $460,7 \pm$ & $455,4 \pm$ & $341,8 \pm$ & $298,6 \pm$ \\
& $56,95 \mathrm{a}$ & $45,39 \mathrm{a}$ & $26 \mathrm{~b}$ & $56,75 \mathrm{~b}$ \\
& $10,81 \pm$ & $9,17 \pm$ & $50,08 \pm$ & $47,22 \pm$ \\
IA (mL) & $2,5 \mathrm{a}$ & $1,28 \mathrm{a}$ & $14,47 \mathrm{~b}$ & $10,45 \mathrm{~b}$ \\
& $6,55 \pm$ & & & $13,25 \pm$ \\
IR (g) & $0,69 \mathrm{a}$ & $7,3 \pm 1,72 \mathrm{a}$ & $15,24 \pm 2 \mathrm{~b}$ & $2,3 \mathrm{~b}$ \\
& & & \\
Gl (mg & $180,7 \pm$ & $167,2 \pm$ & $408 \pm 13,4$ & $406,6 \pm$ \\
dL-1) & $17,32 \mathrm{a}$ & $14,47 \mathrm{a}$ & $\mathrm{b}$ & $6,61 \mathrm{~b}$ \\
GF (mg & $179,8 \pm$ & $166,2 \pm$ & $420,1 \pm$ & $413,6 \pm$ \\
dL-1) & $19,29 \mathrm{a}$ & $17,06 \mathrm{a}$ & $8,22 \mathrm{~b}$ & $49,8 \mathrm{~b}$ \\
\hline
\end{tabular}

Médias seguidas por letras diferentes na mesma linha são estatisticamente diferentes, de acordo com o teste de Tukey $(\mathrm{p}<0,05)$.

A glicemia inicial dos ratos dos grupos $\mathrm{N}$ e NEC foi significativamente menor quando comparada aos ratos dos grupos D e DEC, sendo que esse resultado também foi observado em relação à glicemia final (Tabela 1).

A área dos ácinos glandulares mostrou-se reduzida $(\mathrm{p}<0,05)$ nos ratos dos grupos $\mathrm{D}\left(1368,2 \pm 26,99 \mu \mathrm{m}^{2}\right)$ e DEC $\left(1708,6 \pm 32,68 \mu \mathrm{m}^{2}\right)$ em relação ao grupo $\mathrm{N}$ $\left(2133,4 \pm 40,01 \mu \mathrm{m}^{2}\right)$. Entretanto, não foi verificada diferença significativa entre os grupos N e NEC $(2097,06$ $\left.\pm 38,04 \mu \mathrm{m}^{2}\right)$ (Figura 1). 


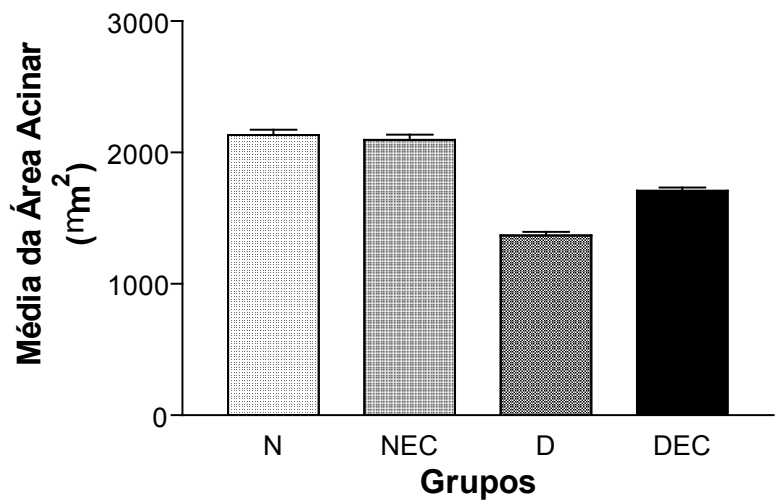

Figura 1: Área acinar média $\left(\mu \mathrm{m}^{2}\right)$ da glândula parótida dos grupos normoglicêmico $(\mathrm{N})$, normoglicêmico suplementado com vitaminas E e C (NEC), diabético (D) e diabético suplementado com vitaminas E e C (DEC).

\section{Conclusão}

A suplementação com as vitaminas $\mathrm{E}$ e $\mathrm{C}$ teve uma repercussão positiva no parênquima da glândula parótida de ratos com diabetes crônico, o que foi evidenciado pela maior densidade acinar, bem como pelo fato de evitar a atrofia das células acinares. Contudo, não se verificaram alteraçóes nos parâmetros fisiológicos de ratos com ou sem suplementação com as vitaminas E e C na comparaçáo entre os grupos normoglicêmicos e entre os grupos diabéticos.

\section{Agradecimentos}

Agradecemos a Maria Angela Moreira, Maria Euride do Carmo Cancino e Maria dos Anjos Fortunato pelo auxílio laboratorial. Este trabalho foi apoiado pelo PIBIC/CNPq- Fundação AraucáriaUEM, Brasil.

\section{Referências}

1. Souza RR, Castro RD, Monteiro CH, Silva SC, Nunes AB. O paciente odontológico portador de diabetes mellitus. Pesq Bras Odontoped Clin Integr. 2003;3:71-7.

2. Taylor GW, Borgnakke WS. Periodontal Disease: associations with diabetes, glycemic control and complications. Oral Dis. 2008;14:191-203.

3. Anderson LC. Parotid gland function in streptozotocin diabetic rats. J Dent Res. 1987;66(2):425-9.

4. Watanabe M, Yamagishi-Wang H, Kawaguchi M. Lowered susceptibility of muscarinic receptor involved in salivary secretion of streptozotocin-induced diabetic rats. Jpn J Pharmacol. 2001;87:117-24.
5. Whitcher D, Sakai N, Williams B, Rahimian R, Anderson L. Gender differences in the effects of streptozotocin-induced diabetes on parasympathetic vasodilation in the rat submandibular gland. Arch Oral Biol. 2010;55:745-53.

6. Mednieks MI, Szczepanski A, Clark B, Hand AR. Protein expression in salivary glands of rats with streptozotocin diabetes. Int JexpPathol. 2009;90(4):412-22.

7. Negrato CA, Tarzia O. Buccal alterations in diabetes mellitus. Diabetology \& Metabolic Syndrome. 2011;2:3.

8. Obrosova IG, Van Huysen C, Fathallah L, Cao X, Greene DA, Stevens MJ. An aldose reductase inhibitor reverses early diabetes-induced changes in peripheral nerve function, metabolism, and antioxidative defense. FASEB Journal. 2002;16:123-5.

9. Sivakumar S, Palsamy P, Subramanian SP. Impact of d-pinitol on the attenuation of proinflammatory cytokines, hyperglycemia-mediated oxidative stress and protection of kidney tissue ultrastructure in streptozotocin-induced diabetic rats. Chemico-Biological Interactions. 2010;188:237-45.

10. Leite, MF, Nicolau J. Sodium Tungstate on Some Biochemical Parameters of the Parotid Salivary Gland of Streptozotocin-Induced Diabetic Rats: A Short-Term Study. Biol Trace Elem Res. 1999;127(2):154-91.

11. Turner S, Zettler G, Arcos MLB, Cremaschi G, Davicino $\mathrm{R}$, Anesini C. Effect of streptozotocin on reactive oxygen species and antioxidant enzyme secretion in rat submandibulary glands: a direct and an indirect relationship between enzyme activation and expression. European Journal of Pharmacology. 2011;659:281-8.

12. Debier C, Larondelle Y. Vitamins A and B: metabolism, roles and transfer to offspring. British Journal of Nutrition. 2005;93:153-74.

13. Meydani M. Vitamin E. The Lancet. 1995;8943(345):170-5.

14. Fadupin GT, Akpoghor AU, Okunade KA. A comparative study of serum ascorbic acid level in people with and without type 2 diabetes in Ibadan, Nigeria. African Journal of Medicine and Medical Sciences. 2007;36(4):335-9.

15. Ardekani MA, Ardekani AS. Effect of vitamin C on blood glucose, serum lipids \& serum insulin in type II diabetes patients. Indian Journal of Medical Research. 2007;126(5):471-4.

16. Bergmeyer HU, Bernt E. Determination of glucose-oxidase and peroxidase. In: Methods of enzymatic analysis. New York: Verlag Chemie-Academic Press; 1974. 1205-12.

17. Furlan MMDP, Molinari SL, Miranda-Neto MH. Resposta dos neurônios mioentéricos do duodeno de ratos ao diabetes de curto prazo. Arq Ciênc Saúde Unipar. 2004;8:95-9.

18. Genuth S. Hormônios das ilhotas pancreáticas. In: Berne RM, Levy MN, editores. Fisiologia. Rio de Janeiro: Guanabara Koogan; 2000. p. 774-97.

19. Furlan MMDP, Molinari SL, Miranda-Neto MH. Morphoquantitative effect of acute diabetes on the proximal colon of adult rats. Arq Neuropsiquiatr. 2002;60:576-81. 
20. Carlton WW, McGavin MD. Patologia veterinária especial de Thomson. 2a ed. Porto Alegre: Artmed; 1998.

21. Cotran RS, Kumar V, Robbins SL. Patologia estrutural e funcional. 5a ed. Rio de Janeiro: Guanabara Koogan; 1996.

22. Caldeira EJ, Camilli JA, Cagnon VHA. Stereology and Ultrastructure of the Salivary Glands of Diabetic Nod Mice Submitted to Long-Term Insulin Treatment. The Anatomical Record Part A. 2005;286A:930-7.

23. Kamata M, Shirakawa M, Kikuche K, Matsuoka T, Aiyama $\mathrm{S}$. Histological analysis of the sublingual gland in rats with streptozotocin-induced diabetes. Okajimas Folia Anat. 2007;84(2):71-6.

24. High AS, Sutton J, Hopper AH. A morphometric study of submandibular salivary gland changes in streptozotocin-induced diabetic rats. Archives of Oral Biology. 1985;9(30):667-71.

25. Isola M, Cossu M, Diana M, Isola R, Loy F, Solinas P, et al. Diabetes reduces statherin in human parotid: immunogold study and comparison with submandibular gland. Oral Diseases. 2012;18:360-4.

26. Hand AR, Weiss RE. Effects of StreptozotocinInduced Diabetes on the Rat Parotid Gland. Lab Invest. 1984;51(4):429-38.

27. Anderson LC, Garrett JR, Thulin A, Proctor GB. Effects of streptozotocin diabetes on sympathetic and parasympathetic stimulation of parotid salivary gland function in rats. Diabetes. 1988;37(4):441-5.

28. Chengxin S, Yan C, Xinzhi L, Guihua T, Yuying F, Yifa Z. Anti-hyperglycemic and anti-oxidative activities of ginseng polysaccharides in STZ-induced diabetic mice. Food Funct. 2014;5:845.

29. Prarabdh CB, Nitin NP, Gauri AC, Telang AG, Sharma AK. Fipronil induced oxidative stress in kidney and brain of mice: protective effect of vitamin $\mathrm{E}$ and vitamin C. Pesticide Biochemistry and Physiology. 2015;118:10-8.

30. Aksoy N, Vural H, Sabuncu T, Arslan O, Aksoy S. Beneficial effects of vitamins $\mathrm{C}$ and $\mathrm{E}$ against oxidative stress in diabetic rats. Nutrition Research. 2005;25:625-30.

31. Anderson LC, Suleiman AH, Garrett JR. Morphological effects of diabetes on the granular ducts and acini of the rat submandibular gland. Microsc Res Tech. 1994;27(1):61-70.

32. Noorafshan A. Volume-weighted mean volume of the submandibular gland acini in male and female diabetic rats. Micron. 2006;37(7):613-6.

33. Vincent AM, Russell JW, Low P, Feldman EL. Oxidative stress in the pathogenesis of diabetic neuropathy. Endocr Rev. 2004;25(4):612-28.

34. Rahimi R, Nikar S, Larijani B, Abdollahi M. A review on the role of antioxidants in the management of diabetes and its complications. Biomed Pharmacother. 2005;59:365-73.

\section{Como citar este artigo:}

Alves AMP, Alves EPB, Mello JM, Zanoni JN, Bespalhok DN, Moreira CR, Ferrari F. Avaliação dos efeitos da suplementação com vitaminas $\mathrm{E}$ e $\mathrm{C}$ sobre os ácinos da glândula parótida de ratos diabéticos crônicos: análise morfológica e quantitativa. Rev. Aten. Saúde. 2016;14(47): 31-35. 